POLIBETÁNICA

Núm. 39, pp. 1-19. México, 2015

\title{
COMPOSICIÓN Y ESTRUCTURA ARBÓREA DE PETENES EN LA RESERVA DE LA BIOSFERA DE LOS PETENES, CAMPECHE, MÉXICO
}

\section{ARBOREAL COMPOSITION AND STRUCTURE OF THE PETENES OF THE PETENES BIOSPHERE RESERVE, CAMPECHE, MEXICO}

\author{
Pedro Zamora-Crescencio ${ }^{1}$, Jean-François Mas ${ }^{2}$, Víctor Rico-Gray ${ }^{3}$, \\ María del Rosario Domínguez-Carrasco ${ }^{1}$, Pascale Villegas ${ }^{1}$, Celso Gutiérrez-Báez ${ }^{1}$, \\ y Roberto Carlos Barrientos-Medina ${ }^{4}$ \\ ${ }^{I}$ Centro de Investigaciones Históricas y Sociales, Universidad Autónoma de Campeche \\ ${ }^{2}$ Centro de Investigación en Geografía Ambiental, Universidad Nacional Autónoma de \\ México. \\ ${ }^{3}$ Instituto de Neuroetología, Universidad Veracruzana, Xalapa, Veracruz 91190, México \\ ${ }^{4}$ Cuerpo Académico de Ecología Tropical, Depto. de Ecología. Facultad de Medicina \\ Veterinaria y Zootecnia, Campus de Ciencias Biológicas y Agropecuarias, Universidad \\ Autónoma de Yucatán. Km. 15.5, Carretera Mérida-Xmatkuil. Mérida, Yucatán, México. \\ Correo electrónico: yecver@hotmail.com
}

\section{RESUMEN}

Analizamos la composición y estructura de la vegetación de 11 petenes de la Reserva de la Biosfera Los Petenes, Campeche. En cada petén seleccionamos cinco parcelas de $20 \mathrm{~m} \times 20 \mathrm{~m}\left(400 \mathrm{~m}^{2}\right.$, total de $\left.2000 \mathrm{~m}^{2}\right)$ donde registramos todos los individuos leñosos con un diámetro a la altura del pecho $(D A P) \geq 5 \mathrm{~cm}$. Se describe la estructura de cada petén con base en los valores relativos de densidad, frecuencia y dominancia (VIR), así como la distribución de clases diamétricas. Se estimó la diversidad por petén usando el índice de Shannon-Wiener $\left(H^{\prime}\right)$. Se estimó la similitud florística entre petenes usando el índice de Morisita. Registramos 2927 individuos de 32 especies leñosas, en 29 géneros y 23 familias. Las familias con mayor número de géneros y especies fueron Fabaceae (3/3) y Sapotaceae $(3 / 3)$. Entre las especies que pre- sentaron mayor densidad están Metopium brownei, Manilkara zapota y Laguncularia racemosa. La distribución en clases de frecuencia usando el DAP, muestra que la mayoría de los individuos se agrupan en las clases inferiores. Con base en el VIR, las especies más importantes en la vegetación de los petenes son: Laguncularia racemosa (128.43), Metopium brownei (128.34) y Manilkara zapota (110.89). Con base en la similitud de especies, el área se dividió en dos grupos: Grupo Centro (petenes 5-11) y Grupo Norte (petenes 1-4). Los petenes 1 y 3 (Grupo Norte) fueron similares entre sí (98\%), mientras que los petenes 5 y 7 (94\%) lo fueron para el Grupo Centro. Los valores de diversidad mostraron que el petén 5 fue el más diverso $\left(H^{\prime}=3.54\right.$, Grupo Centro) y el petén $1\left(H^{\prime}=2.44\right.$, Grupo Norte). Independientemente del grado de perturbación, las especies leñosas características de los 11 petenes fueron 
Metopium brownei y Manilkara zapota y la palma Sabal yapa.

Palabras clave: diversidad, florística, manglar, selva, similitud.

\begin{abstract}
We analyzed the composition and vegetation structure of 11 petenes of the Reserva de la Biosfera de Los Petenes (RBLP), Campeche. In each peten we selected five $20 \mathrm{~m} \times 20 \mathrm{~m}$ plots $\left(400 \mathrm{~m}^{2}\right.$, total $\left.2000 \mathrm{~m}^{2}\right)$ where we recorded and measured all woody individuals with a diameter at breast height $(\mathrm{DBH}) \geq 5 \mathrm{~cm}$. The structure of each peten was described based on relative values of density, frequency and dominance (RIVI), and the distribution of diameter classes. Species diversity for each peten was estimated using the Shannon-Wiener Index (H'), and floristic similarity between petenes was evaluated using the Morisita Index. We recorded 2927 individuals of 32 woody species, in 29 genera, and 23 families. The families with most genera and species were the Fabaceae (3/3) and the Sapotaceae (3/3), while Metopium brownei, Manilkara zapota and Laguncularia racemosa presented the greatest density per hectare. The distribution of diameter classes shows that most individuals were grouped in the lower classes. The highest RIVI was obtained for Laguncularia racemosa (128.43), Metopium brownei (128.34) and Manilkara zapota (110.89). Based on species similarity, the area was divided into two groups: Center Group (petenes 511) and Northern Group (petens 1-4). The highest diversity indices were recorded for peten 5 (Center Group, $\mathrm{H}^{\prime}=3.54$ ), and peten $1\left(\mathrm{H}^{\prime}=2.44\right.$, Northern Group). In the Northern Group, petens 1 and 3 were the
\end{abstract}

most similar (98\%), whereas petens 5 and 7 were the most similar in the Center Group (94\%). Despite the degree of disturbance of the areas, the characteristic woody species were Metopium brownei, Manilkara zapota and the palm Sabal yapa.

Key words: diversity, floristic, mangrove, jungle, similarity.

\section{INTRODUCCIÓN}

La Reserva de la Biosfera de Los Petenes (RBLP, Diario Oficial de la Federación, 1999) con un área ca. 282857 ha (CONANP, 2006) constituye uno de los humedales más particulares del país (Rico-Gray, 1982). Es considerada, además, una región del estado de Campeche de gran importancia, no sólo por que alberga gran diversidad de flora y fauna (Torrescano-Valle, 2010), sino que también por que brinda alimento, agua y protección a muchas especies que la ocupan de forma temporal o permanentes (Rico-Gray et al., 1988; Tun-Dzul y Durán, 2010).

La vegetación de la RBLP está conformada por varias comunidades vegetales. Por ejemplo, manglar en sus diferentes modalidades (manglar de franja, manglar achaparrado), tular (Typha dominguensis), zacatal (Cladium jamaicense), selva baja inundable, blanquizales (Ruppia marítima), chechenales (Metopium brownei) y "petenes" (Rico-Gray, 1982; Trejo-Torres et al., 1993; Zamora-Crescencio, 2003). Los petenes, definidos como islas de vegetación arbórea (Barrera, 1982); están compuestos por elementos florísticos del manglar y de la selva y constituyen la característica más notable de la reserva. Se encuentran inmersos en la zona de manglares, y presentan 
una importante variabilidad en cuanto a su extensión y forma (Mas y Correa, 2000) y la fisonomía de su vegetación (Barrera, 1982; Rico-Gray, 1982; Durán, 1995; Mas y Correa, 2000). La existencia, composición y desarrollo estructural de los petenes está estrechamente relacionada con el relieve y las características del suelo (RicoGray, 1982; Durán, 1987a; Rico-Gray y Palacios-Rios, 1996; White y Hood, 2004; Vargas-Rodríguez et al., 2005), perturbaciones antropogénicas (Koyoc-Ramírez, 2011) y fenómenos naturales (e.g., vientos fuertes, huracanes). La combinación de éstos últimos, puede ocasionar cambios en la estructura de la vegetación, en la diversidad de las especies (Ramírez-Marcial et al., 1998; Sánchez-Aguilar et al., 1999; Von-Gadow et al., 2004), en el número de árboles con diámetros pequeños (Brown y Lugo, 1990) y en la abundancia de especies (William-Linera, 2002).

La CONANP (2006) estima que en la RBLP habitan 678 especies de plantas de 404 géneros y 103 familias de plantas vasculares distribuidas en las diferentes comunidades vegetales. Sin embargo, el conocimiento ecológico de algunas de éstas (e.g., petenes) es aún parcial en cuanto a composición y estructura (diversidad beta). Los primeros estudios cualitativos sobre vegetación y florística en la RBLP se iniciaron a comienzos de los años 1980 (Barrera, 1982; Rico-Gray, 1882; Durán, 1987b). De manera simultánea se iniciaron estudios cuantitativos (Durán, 1987a, 1995; López-Portillo et al., 1989; Koyoc-Ramírez, 2011). Recientemente, se realizó la descripción de la vegetación de la reserva dentro de los límites del municipio de Tenabo (Zamora-Crescencio, 2003), y se resaltó que forman un paisaje naturalmente fragmentado, con una importante variabilidad en los patrones de tamaño, forma y aislamiento (Mas y Correa, 2000). Por otra parte, es notoria la falta de estudios sobre el conocimiento básico de la estructura y composición florística de los petenes localizados dentro de la RBLP. Considerando el tamaño de la reserva, la predominancia de los petenes y la variación que presenta, resulta fundamental realizar estudios sobre la composición florística, la riqueza, estructura y diversidad de las especies vegetales leñosas. Este estudio se centró en describir la composición y estructura de la vegetación leñosa de 11 petenes presentes en la Reserva de la Biósfera de Los Petenes, Campeche, Campeche.

\section{MATERIAL Y MÉTODO}

\section{Descripción del área de estudio}

La Reserva de la Biosfera Los Petenes está ubicada en la franja costera del noroeste del estado de Campeche $\left(20^{\circ}\right.$ y $21^{\circ} \mathrm{N}$ y $90^{\circ} 20^{\prime} \mathrm{y} 90^{\circ} 30^{\prime} \mathrm{W}$; altitud 0-0.4 m) (fig. 1) (Torrescano-Valle, 2010), en los municipios de Calkiní, Hecelchakan, Tenabo y Campeche. Es una gran ciénaga que forma parte de un continuo de humedales en zonas cársticas (Palacio et al., 2005). La reserva ocupa un área de ca. 282,857 ha (CONANP, 2006), y contiene petenes de selva mediana subperennifolia y de manglar, formando un mosaico de asociaciones dentro del gradiente mar-tierra (Rico-Gray; 1982, Durán, 1995). La topografía presenta un relieve muy suave, con porciones bajas o planas, donde predominan los manglares. Geológicamente la reserva está formada por rocas sedimentarias cretácicas (INEGI, 1985a). Los suelos son de origen orgánico y profundos, muy jóvenes y saturados de agua (Tun- 


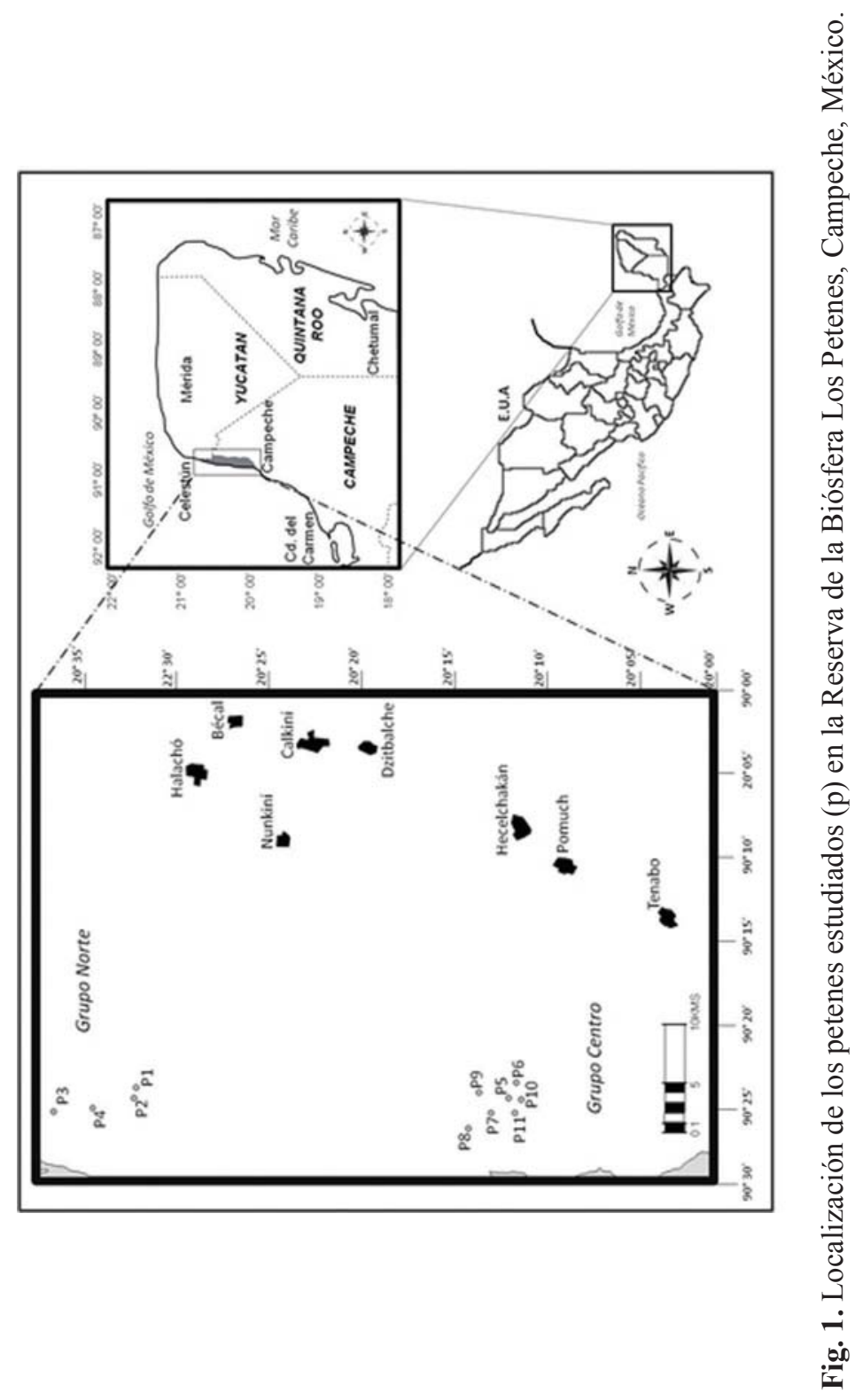


Dzul y Durán, 2010), el suelo predominante es el gleysol mólico, teniendo como suelos secundarios desde solonchac órtico al regosol calcárico (INEGI, 1985b). La ciénega se caracteriza por una red de canales creados por el hombre (siglo XIX) que comunican a varios petenes entre sí y a éstos con el mar (Barrera, 1982; Rico-Gray, 1982). Por los canales fluye un gran volumen de agua dulce proveniente de los ojos de agua o cenotes encontrados en muchos de los petenes (Rico-Gray, 1982). El clima es cálido subhúmedo con régimen de lluvia en verano. La temperatura media anual fluctúa entre los 26 y $28^{\circ} \mathrm{C}$ y la precipitación total anual oscila entre 1000 y $1200 \mathrm{~mm}$ (RicoGray y Palacios-Ríos, 1996). Las especies arbóreas más comunes de los petenes son Rhizophora mangle, Avicennia germinans, Laguncularia racemosa, Manilkara zapota, Ficus continifolia, Swietenia macrophylla, Tabebuia rosea, Sabal yapa, Bravaisia berlandieriana, Metopium brownei, Bursera simaruba, Annona glabra, Pisonia aculeata y Acrostichum aureum (Durán, 1995; Tun-Dzul y Durán, 2010).

\section{Trabajo de campo}

El muestreo de la vegetación se realizó entre diciembre de 1998 y agosto de 1999 $\mathrm{y}$, por su accesibilidad, se analizaron 11 petenes. Los petenes 1 al 4 se ubican en El Remate (Nunkiní, Campeche) al norte de la RBLP (en adelante, Grupo Norte) y los petenes 5 al 11 se ubican a la altura de Isla Jaina al centro de la RBLP (en adelante, Grupo Centro). En el Grupo Norte predominan los elementos del manglar en tanto que en el Grupo Centro las especies de selva (fig. 1). Para la selección de los sitios de muestreo en cada petén se evitaron áreas perturbadas (basada en la presencia/ausencia de tocones). En cada petén se seleccionaron cinco parcelas de $20 \mathrm{~m} \times 20 \mathrm{~m}\left(400 \mathrm{~m}^{2}\right.$, total $\left.2000 \mathrm{~m}^{2}\right)$. En cada parcela se consideraron todos los individuos leñosos con diámetros a altura del pecho $(\mathrm{DAP}=1.3 \mathrm{~m}) \geq 5 \mathrm{~cm}$ (no se consideró la altura de los individuos). Se determinó cada individuo y los ejemplares se depositaron en el herbario (UCAM) del Centro de Investigaciones Históricas y Sociales de la Universidad Autónoma de Campeche, Campeche, México.

El análisis cuantitativo de la estructura de la vegetación leñosa se basó en la densidad, área basal y frecuencia (clases diamétricas). Estas medidas se utilizaron para obtener el valor de importancia relativo (VIR) de cada especie y por petén $(\mathrm{VIR}=$ densidad relativa + frecuencia relativa + dominancia relativa-área basal) (Mueller-Dombois y Ellenberg, 2002). Así mismo, se estimó la diversidad de especies de los 11 petenes con el índice de Shannon-Wiener (H'), ya que refleja la relación entre riqueza y el índice de equitatividad (uniformidad) (Magurran, 1988). En particular, H' refleja a las especies raras, lo cual podría ser de importancia en cuestiones de conservación. El dendrograma obtenido (clasificación) se evaluó a través del coeficiente de correlación cofenética (CCC) y la consistencia de los grupos formados se estimó por medio de un análisis bootstrap, usando 5000 aleatorizaciones, con el programa PAST (Hammer et al., 2001).

\section{RESUlTADOS}

En los 11 petenes estudiados, registramos 2917 individuos de 32 especies, en 29 géneros, 23 familias (Apéndice 2). La variabilidad en la composición numérica 
de familias, especies e individuos y otras características se presenta en el cuadro 1. Las familias mejor representadas fueron la Fabaceae y la Sapotaceae con tres especies cada una $(9.4 \%)$, estas familias también fueron las que más géneros presentaron (3). Las familias anteriores, más Annonaceae y Meliaceae, agrupan al 34.49\% de los géneros encontrados. Los géneros con mayor número de especies (2) fueron Sabal (Arecaceae), Diospyros (Ebenaceae) y Ficus (Moraceae). Sólo tres especies, Metopium brownei, Manilkara zapota y Sabal yapa representaron el 9.3\% del total de especies registradas (cuadro 2).

Al considerar el dendrograma de similitud (fig. 3), y tomando en cuenta como umbral el $40 \%$ de similitud florística, se divide claramente a los petenes por grupo: Norte y Centro. Los petenes más afines del Grupo Norte fueron el 1 y el 3 (98\%), en tanto los más similares en el Grupo Centro fueron el 5 y el 7 (94\%).

La composición florística del Grupo Norte fue de 16 familias, 18 géneros y 19 especies. El petén 1 tuvo mayor número de especies (13) y el petén 2 el menor número (7). El petén 4 exhibió la mayor abundancia (253 individuos) (cuadro 1). La diversidad de especies fue muy similar entre petenes $\left(\mathrm{H}^{\prime}=2.05-2.44\right) \mathrm{y}$, por consiguiente, los valores de equitatividad (cuadro 1).

La composición florística del Grupo Centro fue de 20 familias, 25 géneros y 27 especies. Los petenes 8 y 11 presentaron la mayor riqueza de especies (15 especies), mientras que los petenes 8 y 5 presentaron la mayor abundancia (394 y 376 individuos) (cuadro 1). El índice de diversidad también varió entre petenes (cuadro 1); La mayor diversidad se encontró en el petén $5\left(\mathrm{H}^{\prime}=3.54\right)$ y la menor en los petenes 8 y 6 ( $\mathrm{H}^{\prime} 2.54$ y 2.61. Los petenes 5,7 y 10 fueron los más uniformes (cuadro 1). El petén 5 fue el mejor balanceado en número de especies/abundancia.

El Grupo Norte agrupó a un menor número de individuos (797), donde el petén 4 fue el más denso (1 $265 \mathrm{ind} / \mathrm{ha}$ ) y el menos denso fue el petén 2 (625 ind/ha). La mayor densidad por especie fue para Laguncularia racemosa (1735 ind/ha) y para Rhizophora mangle (1 $010 \mathrm{ind} / \mathrm{ha}$ ), la menor densidad fue para Manilkara zapota (385 ind/ha) y para Sabal yapa (345 ind/ha). Estas cuatro especies constituyeron el 87.2\% (695 individuos) de los individuos considerados. La estructura de los petenes 1, 3 y 4 fue una curva en forma de J-invertida, mientras que el petén 2 presentó más individuos en las clases 2 y 3 (fig. 2). El mayor número de individuos $(>100)$ en la clase 1 fue de Laguncularia racemosa y de Rhizophora mangle. La mayor clase diamétrica $(>55$ $\mathrm{cm})$ se presentó en el petén 2 , donde 26 individuos de Laguncularia racemosa presentaron un DBH de hasta $89 \mathrm{~cm}$. Las especies ubicadas en la clase diamétrica mayor (>55 cm) fueron Laguncularia racemosa, Manilkara zapota y Sabal yapa.

El Grupo Centro agrupó a más individuos (2 130) y el petén 8 fue el más denso (1970 ind/ha), mientras que los petenes $6,9 \mathrm{y}$ 11 fueron los menos densos $(1015,1165$ y $1395 \mathrm{ind} / \mathrm{ha})$. La especie con mayor densidad fue Metopium brownei (3 775 $\mathrm{ind} / \mathrm{ha}$ ) y las densidades más bajas correspondieron a Piscidia piscipula y Bravaisia berlandieriana (495 y $295 \mathrm{ind} / \mathrm{ha}$ ). Estas tres especies la mayor densidad, mientras que el resto se distribuye en 22 especies. 
Zamora-Cresencio, P. et al.: Composición y estructura arbórea de petenes, Reserva de la Biosfera Los Petenes, Camp., Méx.

Cuadro 1. Características de la vegetación de 11 petenes estudiados.

\begin{tabular}{|c|c|c|c|c|c|c|}
\hline Petén & Familias & Especies & Individuos & Diversidad & Equitatividad & $\begin{array}{c}\text { Área basal } \\
\left(\mathrm{m}^{2} / \mathrm{ha}\right)\end{array}$ \\
\hline \multicolumn{7}{|c|}{ Grupo Norte } \\
\hline 1 & 12 & 13 & 190 & 2.44 & 0.66 & 52.30 \\
\hline 2 & 7 & 7 & 125 & 2.05 & 0.73 & 75.71 \\
\hline 3 & 12 & 12 & 229 & 2.06 & 0.62 & 34.26 \\
\hline 4 & 7 & 9 & 253 & 2.37 & 0.74 & 24.79 \\
\hline \multicolumn{7}{|c|}{ Grupo Centro } \\
\hline 5 & 10 & 13 & 376 & 3.54 & 0.95 & 37.17 \\
\hline 6 & 13 & 14 & 203 & 2.61 & 0.68 & 46.29 \\
\hline 7 & 12 & 13 & 307 & 3.30 & 0.90 & 32.51 \\
\hline 8 & 13 & 15 & 394 & 2.54 & 0.65 & 23.35 \\
\hline 9 & 12 & 14 & 233 & 2.82 & 0.74 & 35.74 \\
\hline 10 & 10 & 12 & 338 & 3.30 & 0.92 & 25.54 \\
\hline 11 & 11 & 15 & 279 & 2.78 & 0.71 & 48.49 \\
\hline
\end{tabular}

En cuanto a su estructura, la mayoría de los petenes (exceptuando 6 y 9) en este grupo se presenta como una J-invertida (fig. 2). Las especies que aportaron mayor cantidad de individuos $(>100)$ en la primera clase diamétrica fueron Metopium brownei, Gymnanthes lucida, Manilkara zapota y Bursera simaruba. La mayor clase diamétrica $(\geq 55 \mathrm{~cm})$ fue más común en el petén 6 (hasta $>70 \mathrm{~cm}$ de DBH). En general, las especies ubicadas en la mayor clase diamétrica $(\geq 55 \mathrm{~cm})$ fueron Manilkara zapota, Metopium brownei y Piscidia piscipula.

En el Apéndice 1 se muestran por petén las especies que tuvieron mayor VIR. Por ejemplo, en el Grupo Norte, Laguncularia racemosa presentó en mayor VIR por especie en el petén 2. Manilkara zapota presentó el segundo VIR por especie en el petén 2. En el Grupo Centro, la especie con mayor VIR fue Metopium brownei, la segunda especie con el VIR más alto fue M. zapota.

\section{DISCUSIÓN}

Los resultados sugieren que la presencia de las diferentes especies está asociada con factores físicos (e.g., microtopografía), periodos de inundación y químicos del suelo, ya que una diferencia de unos pocos centímetros del nivel del suelo puede determinar a las diferentes comunidades vegetales (Durán, 1987a; López-Portillo et al., 1989; Rico-Gray y Palacios-Rios, 1996; Flores-Verdugo et al., 2007; Moreno-Casasola et al., 2010). La riqueza de especies en los petenes analizados varió entre siete y 15 especies, con los valores más altos en los petenes 8 y 11 . La riqueza de especies (32 especies) fue menor a la 


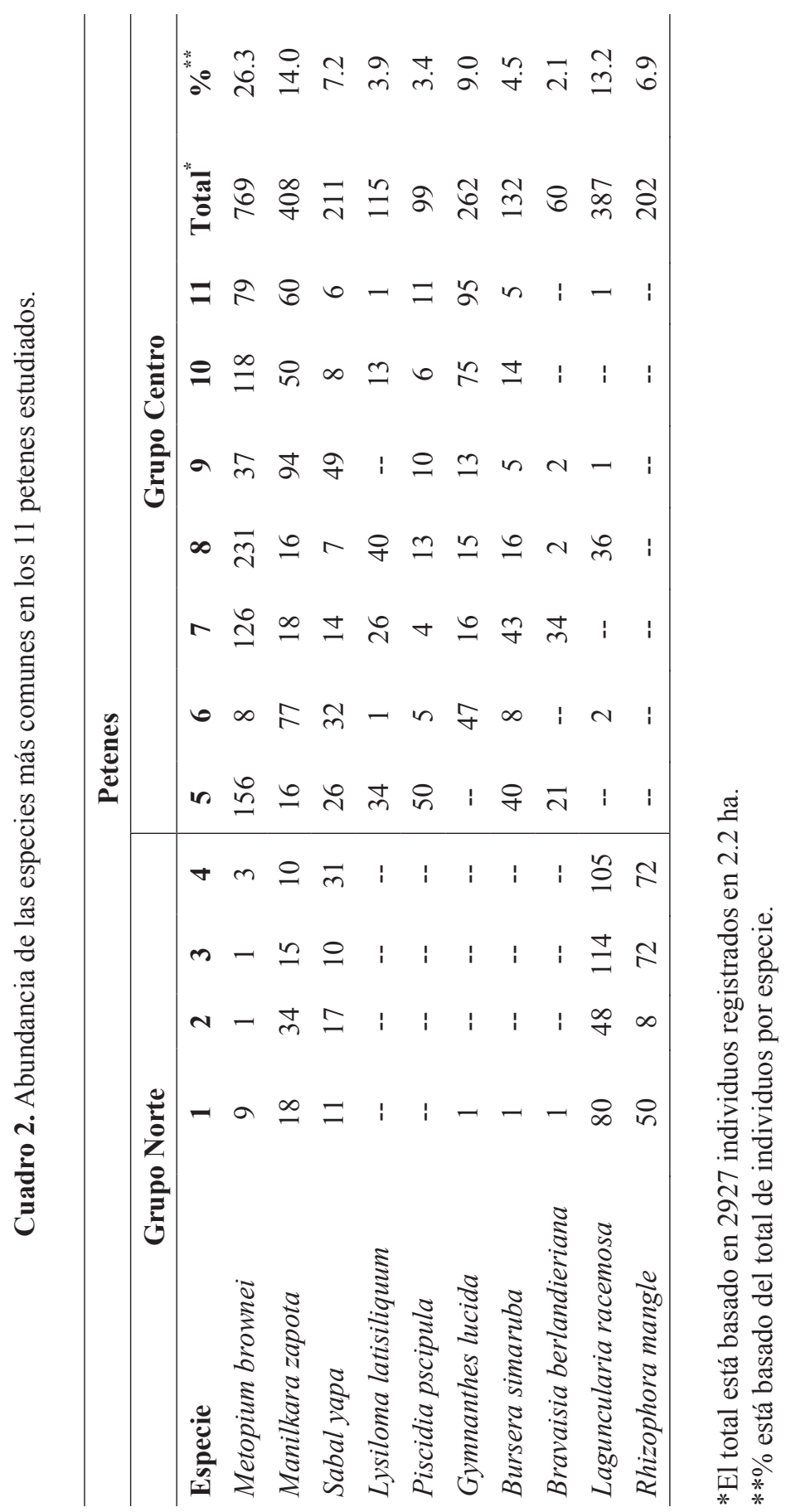




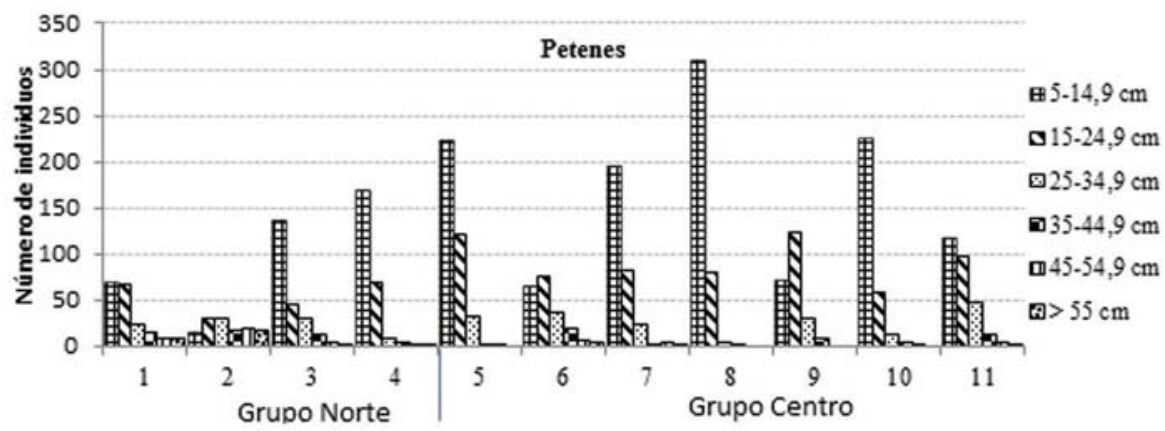

Fig. 2. Distribución de las clases diamétricas de los individuos con DAP $\geq 5 \mathrm{~cm}$ de $\operatorname{los}$ once petenes estudiados.

reportada (316 especies Rico-Gray, 1982; y 153 especies Durán, 1987b). Sin embargo, en nuestro estudio sólo se tomaron en cuenta a los petenes y no a la vegetación circundante (e.g., selva baja inundable, tular, zacatales). Por ejemplo, encontramos especies (Mosanonna depressa, Gymnanthes lucida, Elaeodendron trichotomum, Krugiodendron ferreum y Zuelania guidonia) no compartidas por autores previos (Durán, 1995; Rico-Gray, 1982), así que el $43.8 \%$ de las especies registradas no fueron reportadas anteriormente.

Los resultados indican que los valores de diversidad ( $\left.\mathrm{H}^{\prime}\right)$ de los petenes analizados están mayormente ligados con la distribución de abundancias de las especies más que a su riqueza específica. Por ejemplo, los petenes 1 y 5 presentan un valor de H' de = 2.44 y 3.54 respectivamente con el mismo número de especies (cuadro 1). Así mismo y 'por definición' (Magurran, 1988), el valor de H’ está íntimamente asociado con el número de especies raras, lo cual, a su vez, tiene mucho que ver con los criterios usados en esquemas de conservación (ver abajo). Las familias con mayor número de especies (Fabaceae y Sapotaceae) integran al $18.8 \%$ (seis especies) de las especies de la flora de los petenes analizados. Además, Fabaceae es la que presenta el mayor número de especies en otros estudios (e.g., Durán, 1987b; Ibarra-Manríquez et al., 1995; Villaseñor, 2003; Puc-Garrido, 2010), y ambas familias (Fabaceae y Sapotaceae) son las mejor representadas en selvas medianas subperennifolias (Gómez-Pompa, 1977; Bongers et al., 1988; Vázquez-Torres, 1991; Pennington y Sarukhán, 2005). De manera similar a lo reportado por Durán (1987b), Laguncularia racemosa y Rhizophora mangle fueron las especies que presentaron la mayor densidad en el Grupo Norte. Mientras que Metopium brownei y Manilkara zapota lo fueron para el Grupo Centro. M. brownei y M. zapota presentaron el mayor número de individuos para la Región Centro (Durán, 1987a; Koyoc-Ramírez et al., 2011). 
Los resultados indican que la estructura (clases diamétricas) general de las comunidades estudiadas formó una J-invertida en ambos grupos (exceptuando el petén 2 en el Grupo Norte y los petenes 6 y 9 en el Grupo Centro). La forma de J-invertida sugiere que hay mayor reclutamiento lo que conduce a tener una regeneración natural (Christo et al., 2009, pero ver Condit et al., 1998). Así, en el Grupo Norte, el área basal del petén $2\left(75.71 \mathrm{~m}^{2} /\right.$ ha $)$ fue la más alta, ya que presentó un mayor número de árboles gruesos (cuadro 1). Mientras que el área basal de los petenes en el Grupo Centro fue baja, en particular si se le compara con los valores obtenidos por Durán (1987a). Laguncularia racemosa presenta el VIR más alto en el Grupo Norte (Durán 1987a). En el Grupo Centro Manilkara zapota presenta VIR altos (110.89) y se considera como la más importante en los petenes considerados como comunidades de selva. El análisis de similitud mostró mayor similitud estructural entre algunos petenes (fig. 3), por ejemplo, entre los que comparten a Rhizophora mangle y Laguncularia racemosa, a los que comparten a Manilkara zapota y Sabal yapa y a los que comparten a Metopium brownei, Manilkara zapota y Bursera simaruba. Los resultados indican diferencias en la composición florística entre petenes, a pesar de las distancias geográficas, indicando que difiere la estructura de los petenes entre grupos y entre petenes de un mismo grupo.

Finalmente, los petenes no son únicos para el estado de Campeche, también los hay en los estados de Quintana Roo y Yucatán. Sin embargo, el área que abarca la RBLP es considerablemente grande, contiene a la zona con mayor diversidad de petenes (V. Rico-Gray, datos no publicados) y aquí en esta región anidan muchas aves tanto residentes como migratorias (Rico-Gray et al., 1988). Por otra parte, muchos petenes contienen restos arqueológicos (Barrera, 1982) y entre las especies más importantes hay varias consideradas como posiblemente manejadas por el hombre en épocas pasadas (Barrera, 1982; Rico-Gray et al., 1985). Lo anterior hace que esta área sea realmente importante como para ser considerada en planes de manejo y de conservación (Watts y Rico-Gray, 1987).

\section{Conclusiones}

Independientemente del grado de perturbación, de las 32 especies leñosas encontradas, Metopium brownei y Manilkara zapota y la palma Sabal yapa están presentes en los 11 petenes analizados. Las dos primeras especies presentaron mayor densidad de individuos. Por otra parte, resulta curioso, pero las tres especies son consideradas como muy útiles y pudieron verse favorecidas por algún tipo de manejo por los mayas (Barrera, 1982; Rico-Gray, 1982; Rico-Gray et al., 1985). Los petenes con mayor diversidad son los de selva. La mayor similitud entre petenes se encontró en el Grupo Centro, mientras que en el Grupo Norte se presenta como un grupo diferente, básicamente por la presencia y abundancia de Laguncularia racemosa y de Rhizophora mangle, típicas especies de manglar.

\section{AgRadecimientos}

Este estudio fue parte del proyecto Q026 "Fragmentación de hábitats y evaluación de la biodiversidad en la región de los Petenes" financiado por la Comisión Nacional para el Conocimiento y Uso de la Biodiversidad (CONABIO). Al licenciado 
Zamora-Cresencio, P. et al.: Composición y estructura arbórea de petenes, Reserva de la Biosfera Los Petenes, Camp., Méx.

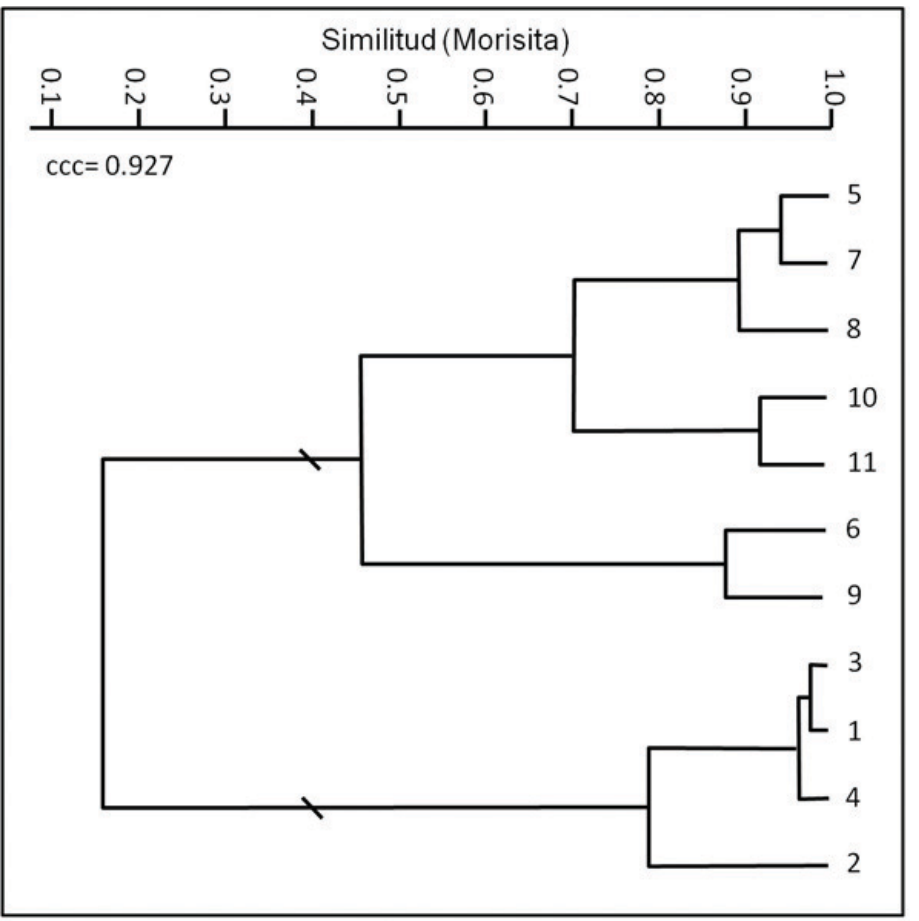

Fig. 3. Dendrograma producido en la clasificación de los datos de abundancia de las especies por peten en la Reserva de la Biosfera de Los Petenes, Campeche.

Juan José Cosgaya Medina por las figuras que se encuentran en el texto, al biólogo Fausto R. del Ángel Tafoya por el apoyo de campo y al doctor William J. Folan por la revisión del abstract.

\section{LITERATURA CITADA}

Angiosperm Phylogeny Group, 2009. “An update of the Angiosperm Phylogeny Group classification for the orders and families of flowering plants AGP III'. Botanical Journal of the Linnean Society, 161: 105-121.
Barrera, A., 1982. "Los petenes del noroeste de Campeche. Su exploración ecológica en perspectiva”. Biótica, 7(2): 163-169.

Bongers, E.J.; J. Pompa, Meave del Castillo, y J. Carabias, 1988. "Structure and floristic composition of the lowland rain forest of the Tuxtlas, México". Vegetation, 74: 55-80.

Brown, S., y A.E. Lugo, 1990. "Tropical secondary forests". Journal of Tropical Ecology, 6: 1-32. 
CONANP, 2006. Programa de conservación y manejo: reserva de la Biosfera Los Petenes. Comisión Nacional de Áreas Naturales Protegidas. 203 pp., México, DF.

Christo, A.G.; R.R. Guedes-Bruni, P. De Araújo, F. Sobrinho, A. Gomes Da Silva, y A. Luna-Peixoto, 2009. "Structure of the shrub-arboreal component of an atlantic forest fragmenton a hillock in the central lowland of Rio de Janeiro, Brazil". Interciencia, 34: 232-239.

Condit, R.; R. Sukumar, S.P. Hubbell, y R.B. Foster, 1998. "Predicting population trends from size distributions: a direct test in a tropical tree community". American Naturalist, 152: 495-509.

Diario Oficial de la Federación (DOF), 1999. Decreto por el que se declara área natural protegida, con el carácter de reserva de la biosfera, la región del estado de Campeche conocida como Los Petenes, con una superficie total de 282857 hectáreas. 24 de mayo de 1999.

Durán, G., R., 1987a. "Descripción y análisis de la estructura y composición de la vegetación de los petenes del noroeste de Campeche, México". Biótica, 12(3): 181-198.

, 1987b. "Lista florística de la región de los petenes, Campeche, México". Biótica, 12(3): 199-208.

, 1995. "Diversidad florística de los petenes de Campeche". Acta Botánica Mexicana, 31: 73-84.
Flores-Verdugo, F.; P. Moreno-Casasola, C. Agraz-Hernández, H. López-Rosas, D. Benítez-Pardo, y A. Travieso-Bello, 2007. "La topografía y el hidroperíodo: dos factores que condicionan la restauración de los humedales costeros". Bol. Soc. Bot. Méx., 80S: 33-47.

Gómez-Pompa, A., 1977. Ecología de la vegetación de Veracruz. INIREB, Xalapa, Veracruz, México. 91 pp.

Hammer, Ø.; D.A.T. Harper, y P.D. Ryan, 2001. PAST: Paleontological statistics software package for education and data analysis. Paleontología electrónica, $9 \mathrm{pp}$.

Ibarra-Manríquez, G.; J.L. Villaseñor, y R. Durán G., 1995. "Riqueza de especies y endemismo del componente arbóreo de la Península de Yucatán, México". Bol. Soc. Bot. Méx., 57: 49-77.

Instituto Nacional de Estadística e Informática (INEGI). 1985a. Carta geológica. "Hoja Calkiní F15-9-12, escala 1: 250 000". INEGI, Aguascalientes, México.

, 1985b. Carta edafológica. "Hoja Calkiní. F15-9-12, escala 1: 250 000". INEGI, Aguascalientes, México.

Judd, W.S.; C.S. Campbell, E.A. Kellog, P.E. Stevens, y M.J. Donoghue, 2008. Plant Systematics: A Phylogenetic Approach. Sinauer Associates, Inc. Publishers Sunderland, Massachusetts, USA, $611 \mathrm{pp}$.

Koyoc-Ramírez, L.G., 2011. "Influencia de la perturbación antropogénica, en la 
vegetación y suelo de petenes de selva de la Reserva de La Biosfera Los Petenes", tesis de maestría, El Colegio de la Frontera Sur. Campeche. 65 pp.

López-Portillo, J.; E. Ezcurra, y J.M. Maass. 1989. "Los petenes de Sian ka'an, Quintana Roo y su relación con gradientes de presión hídrica". Acta Botánica Mexicana, 5: 19-29.

Magurran, A.E., 1988. Ecological Diversity and its Measurement. Princeton, NJ. Princenton University Press.

Mas, J.F., y J. Correa Sandoval, 2000. "Análisis de la fragmentación del paisaje en el Área Protegida Los Petenes, Campeche, México". Investigaciones Geográficas, Boletín del Instituto de Geografia de la Universidad Nacional Autónoma de México, 43: 42-59.

Moreno-Casasola, P.; E. Cejudo-Espinosa, A. Capistrán-Barradas, D. InfanteMata, H. López-Rosas, G. CastilloCampos, J. Pale-Pale, y A. CamposCascaredo, 2010. "Composición florística, diversidad y ecología de humedales herbáceos emergentes en la planicie costera central de Veracruz, México". Boletín de la Sociedad Botánica de México, 87: 29-50.

Mueller-Dombois, D., y H. Ellenberg, 2002. Aims and Methods of Vegetation Ecology. John Willer and Sons. N. York $547 \mathrm{pp}$.

Palacio, A., G.; V. Medina, y F. Bautista, 2005. "Diagnóstico ambiental de la costa del estado de Campeche: enfoques geomorfólogicos y geopedoló- gico", en: F. Bautista, y G. Palacio A. (Eds.). Caracterización y Manejo de los Suelos de la Península de Yucatán: Implicaciones Agropecuarias, Forestales y Ambientales. Universidad Autónoma de Campeche, Universidad Autónoma de Yucatán y Instituto Nacional de Ecología, México, DF, pp. 59-72.

Pennington, T.D., y J. Sarukhán, 2005. Árboles tropicales de México. Manual para la identificación de las principales especies. UNAM-Fondo de Cultura Económica. México. 523 pp.

Puc-Garrido, E.C., 2010. "Estructura y composición florística de la selva mediana subperennifolia de los alrededores de la comunidad de Bethania y del Centro de Investigación de Vida Silvestre de Hampolol (CIVS-Hm) del municipio de Campeche", tesis de licenciatura. Facultad de Ciencias Químico Biológicas. Universidad Autónoma de Campeche. 75 pp.

Ramírez-Marcial, N.; S. Ochoa-Gaona, y M. González-Espinosa, 1998. "Análisis florístico y sucesional en la Estación Biológica cerro Huitepec, Chiapas, México". Acta Botánica Mexicana, 44: 59-85.

Rico-Gray, V., 1982. "Estudio de la vegetación de la zona costera inundable del noroeste del estado de Campeche, México. Los petenes". Biótica, 7: 171-190.

R. Domínguez, y G. Cobb, 1988. "Avifauna de la zona costera inundable del noroeste de Campeche, México: 
lista de especies y su distribución con respecto a la vegetación". Biótica, 13: 81-92.

Rico-Gray, V.; A. Gómez-Pompa, y C. Chan., 1985. "Las selvas manejadas por los mayas de Yohaltún, Campeche". Biótica, 10: 321-328.

Rico-Gray, V., y M. Palacios-Ríos, 1996. "Salinidad y el nivel del agua como factores en la distribución de la vegetación en la ciénega del NW de Campeche, México". Acta Botánica Mexicana, 34: 53-61.

Sánchez-Aguilar, R.L., y S. Rebolar D., 1999. "Deforestación en la Península de Yucatán". Madera y Bosques, 5: 3-17.

Torrescano-Valle, N., 2010. "Estudio de caso: los petenes de Campeche, Reserva de la Biosfera", en: Villalobos-Zapata, G.J., y J. Mendoza Vega (Eds). $L a$ Biodiversidad en Campeche: Estudio de Estado. Comisión Nacional para el Conocimiento y Uso de la Biodiversidad (CONABIO), Gobierno del estado de Campeche, Universidad Autónoma de Campeche y El Colegio de la Frontera Sur (ECOSUR), México, DF, pp. 165-170.

Trejo-Torres, J.C.; R. Durán-G., y I. Olmsted, 1993. "Manglares de la Península de Yucatán", en: S.I. Salazar-Vallejo y N.E. González (Eds). Biodiversidad Marina y Costera de México. Comisión Nacional para el Conocimiento y Uso de la Biodiversidad y Centro de Investigaciones de Quintana Roo, México, DF pp. 660-672.
Tun-Dzul, F., y R. Durán G., 2010. "Estudio de caso: la vegetación de los petenes de Campeche", en: Villalobos-Zapata, G.J., y J. Mendoza Vega (Eds). La Biodiversidad en Campeche: Estudio de Estado. Comisión Nacional para el Conocimiento y Uso de la Biodiversidad (CONABIO), Gobierno del estado de Campeche, Universidad Autónoma de Campeche y El Colegio de la Frontera Sur (ECOSUR), México, DF, pp. 163-164.

Vargas-Rodríguez, Y.L.; J.A. VázquezGarcía, y G. Bruce-Williamson. 2005. "Environmental correlatos of tree and seedling-sapling distributions in a Mexican tropical dry forest". Plant Ecology, 180: 117-134.

Vázquez-Torres, M., 1991. "Flora vascular y diversidad de especies arbóreas del dosel superior (en una muestra de selva alta sobre sustrato cárstico en la zona de Uxpanapa)". Textos Universitarios, Universidad Veracruzana, Xalapa, Veracruz. 184 pp.

Villaseñor, J.L., 2003. "Diversidad y distribución de las Magnoliophyta de México”. Interciencia, 28: 160-186.

Von-Gadow, K.; S. Sánchez-Orois, y O.A. Aguirre-Calderón, 2004. "Manejo forestal con bases científicas". Madera y Bosques, 10: 3-16.

Watts, E.S., y V. Rico-Gray. 1987. "Los primates de la Península de Yucatán, México: estudio preliminar sobre su distribución actual y estado de conservación”. Biotica, 12: 57-66. 
Zamora-Cresencio, P. et al.: Composición y estructura arbórea de petenes, Reserva de la Biosfera Los Petenes, Camp., Méx.

William-Linera, G., 2002. "Tree species richness complementarity, disturbance and fragmentation in a Mexican tropical montane cloud forest". Biodiversity and Conservation, 11: 1825-1843.
Zamora-Crescencio, P., 2003. "Contribución al estudio florístico y descripción de la vegetación del municipio de Tenabo, Campeche, México". Polibotánica, 15: $1-40$.

White, D.A., y C.S. Hood. 2004 “"Vegetation patterns and environmental gradients in tropical dry forest of the northern Yucatan Peninsula". Journal of Vegetation Science, 15: 151-160.

Recibido: 16 agosto 2013. Aceptado: 20 junio 2014. 
Apéndice 1. Parámetros estructurales de once petenes estudiados.

\begin{tabular}{|c|c|c|c|c|}
\hline Especie & $\begin{array}{c}\text { Densidad } \\
\text { relativa }\end{array}$ & $\begin{array}{c}\text { Frecuencia } \\
\text { relativa }\end{array}$ & $\begin{array}{c}\text { Dominancia } \\
\text { relativa }\end{array}$ & $\begin{array}{c}\text { Valor de } \\
\text { importancia }\end{array}$ \\
\hline \multicolumn{5}{|l|}{ Petén 1} \\
\hline Laguncularia racemosa & 42.11 & 17.86 & 61.82 & 121.78 \\
\hline Rhizophora mangle & 26.32 & 10.71 & 17.73 & 54.76 \\
\hline Manilkara zapota & 9.47 & 14.29 & 7.53 & 31.29 \\
\hline Sabal yapa & 5.79 & 10.71 & 8.03 & 24.53 \\
\hline Metopium brownei & 4.74 & 14.29 & 0.72 & 19.74 \\
\hline 8 spp. más & 11.58 & 32.14 & 4.18 & 49.90 \\
\hline \multicolumn{5}{|l|}{ Petén 2} \\
\hline Laguncularia racemosa & 38.40 & 20.83 & 69.19 & 128.43 \\
\hline Manilkara zapota & 27.20 & 20.83 & 13.28 & 61.32 \\
\hline Sabal yapa & 13.60 & 20.83 & 3.43 & 37.86 \\
\hline Ficus obtusifolia & 12.00 & 16.67 & 6.79 & 35.45 \\
\hline Rhizophora mangle & 6.40 & 8.33 & 6.98 & 21.71 \\
\hline 2 spp. más & 2.40 & 12.51 & 0.33 & 15.23 \\
\hline \multicolumn{5}{|l|}{ Petén 3} \\
\hline Laguncularia racemosa & 49.78 & 18.52 & 59.15 & 127.45 \\
\hline Rhizophora mangle & 31.44 & 11.11 & 16.19 & 58.74 \\
\hline Manilkara zapota & 6.55 & 18.52 & 15.58 & 40.65 \\
\hline Sabal yapa & 4.37 & 14.81 & 4.50 & 23.68 \\
\hline Ficus obtusifolia & 1.75 & 7.41 & 1.44 & 10.59 \\
\hline 8 spp. más & 6.11 & 29.63 & 3.14 & 38.89 \\
\hline \multicolumn{5}{|l|}{ Peten 4} \\
\hline Laguncularia racemosa & 41.50 & 19.23 & 37.62 & 98.35 \\
\hline Rhizophora mangle & 28.46 & 15.38 & 10.77 & 54.61 \\
\hline Sabal yapa & 12.25 & 15.38 & 21.31 & 48.95 \\
\hline Tabebuia rosea & 9.09 & 15.38 & 7.64 & 32.11 \\
\hline Manilkara zapota & 3.95 & 7.69 & 19.98 & 31.62 \\
\hline 4 spp. más & 4.75 & 26.94 & 2.68 & 34.36 \\
\hline
\end{tabular}


Apéndice 1. Continuación.

\begin{tabular}{|c|c|c|c|c|}
\hline Especie & $\begin{array}{c}\text { Densidad } \\
\text { relativa }\end{array}$ & $\begin{array}{c}\text { Frecuencia } \\
\text { relativa }\end{array}$ & $\begin{array}{c}\text { Dominancia } \\
\text { relativa }\end{array}$ & $\begin{array}{c}\text { Valor de } \\
\text { importancia }\end{array}$ \\
\hline \multicolumn{5}{|l|}{ Peten 5} \\
\hline Metopium brownei & 41.49 & 10.42 & 37.86 & 89.77 \\
\hline Lysiloma latisiliquum & 9.04 & 10.42 & 22.40 & 41.86 \\
\hline Piscidia piscipula & 13.30 & 10.42 & 11.13 & 34.84 \\
\hline Sabal yapa & 6.91 & 10.42 & 10.60 & 27.93 \\
\hline Bursera simaruba & 10.64 & 10.42 & 6.09 & 27.14 \\
\hline 8 spp. más & 18.62 & 47.90 & 11.92 & 78.46 \\
\hline \multicolumn{5}{|l|}{ Peten 6} \\
\hline Manilkara zapota & 37.93 & 12.82 & 60.14 & 110.89 \\
\hline Gymnanthes lucida & 23.15 & 12.82 & 6.42 & 42.40 \\
\hline Sabal yapa & 15.76 & 12.82 & 9.35 & 37.94 \\
\hline Metopium brownei & 3.94 & 12.82 & 6.30 & 23.06 \\
\hline Bursera simaruba & 3.94 & 7.69 & 7.50 & 19.14 \\
\hline 9 spp. más & 15.28 & 41.03 & 10.29 & 66.57 \\
\hline \multicolumn{5}{|l|}{ Peten 7} \\
\hline Metopium brownei & 41.04 & 10.87 & 41.92 & 93.83 \\
\hline Lysiloma latisiliquum & 8.47 & 10.87 & 16.10 & 35.44 \\
\hline Manilkara zapota & 5.86 & 8.70 & 17.35 & 31.91 \\
\hline Bursera simaruba & 14.01 & 10.87 & 6.49 & 31.36 \\
\hline Bravaisia berlandieriana & 11.07 & 10.87 & 1.62 & 23.57 \\
\hline 8 spp. más & 19.55 & 47.82 & 16.52 & 83.89 \\
\hline \multicolumn{5}{|l|}{ Peten 8} \\
\hline Metopium brownei & 58.63 & 11.90 & 57.81 & 128.34 \\
\hline Lysiloma latisiliquum & 10.15 & 11.90 & 15.20 & 37.25 \\
\hline Laguncularia racemosa & 9.14 & 7.14 & 9.98 & 26.26 \\
\hline Manilkara zapota & 4.06 & 11.90 & 4.68 & 20.65 \\
\hline Bursera simaruba & 4.06 & 9.52 & 2.12 & 15.70 \\
\hline 10 spp. más & 13.96 & 47.64 & 10.21 & 71.80 \\
\hline
\end{tabular}


Apéndice 1. Conclusión.

\begin{tabular}{lcccc}
\hline \multicolumn{1}{c}{ Especie } & $\begin{array}{c}\text { Densidad } \\
\text { relativa }\end{array}$ & $\begin{array}{c}\text { Frecuencia } \\
\text { relativa }\end{array}$ & $\begin{array}{c}\text { Dominancia } \\
\text { relativa }\end{array}$ & $\begin{array}{c}\text { Valor de } \\
\text { importancia }\end{array}$ \\
\hline Peten 9 & & & & \\
Manilkara zapota & 40.34 & 12.50 & 42.91 & 95.75 \\
Sabal japa & 21.03 & 12.50 & 18.03 & 51.56 \\
Metopium brownei & 15.88 & 12.50 & 18.87 & 47.25 \\
Piscidia piscipula & 4.29 & 10.00 & 5.85 & 20.14 \\
Sideroxylon americanum & 3.00 & 12.50 & 3.53 & 19.03 \\
9 spp. más & 15.46 & 40 & 10.81 & 66.27 \\
& & & & \\
Peten 10 & & & & \\
Metopium brownei & 34.91 & 13.89 & 34.83 & 83.63 \\
Manilkara zapota & 14.79 & 11.11 & 23.00 & 48.91 \\
Gymnanthes lucida & 22.19 & 11.11 & 9.30 & 42.60 \\
Avicennia germinans & 8.88 & 8.33 & 6.32 & 23.53 \\
Bursera simaruba & 4.14 & 11.11 & 4.23 & 19.48 \\
7 spp. más & 15.09 & 44.45 & 22.32 & 81.85 \\
& & & & \\
Peten 11 & & & & \\
Metopium brownei & 28.32 & 12.5 & 44.71 & 85.53 \\
Gymnanthes lucida & 34.05 & 12.5 & 13.20 & 59.75 \\
Manilkara zapota & 21.51 & 12.5 & 21.50 & 55.51 \\
Piscidia piscipula & 3.94 & 10 & 9.72 & 23.66 \\
Melicoccus oliviformis & 2.51 & 7.5 & 1.59 & 11.60 \\
ssp. oliviformis & & & & \\
10 spp. más & 9.67 & 45 & 9.28 & 63.95
\end{tabular}


Apéndice 2. Lista florística de los once petenes estudiados. Se elaboró de acuerdo al sistema de clasificación del Grupo para la Filogenia de las Angiospermas (AGP 2009) y Judd et al. 2008.

\section{FAMILIA/ESPECIES}

\section{ACANTHACEAE}

Avicennia germinans (L.) L.

Bravaisia berlandieriana (Nees) T.F. Daniel

\section{ANACARDIACEAE}

Metopium brownei (Jacq.) Urb.

ANNONACEAE

Annona glabra L.

Mosannona depressa (Baill.) Chatrou

APOCYNACEAE

Tabernaemontana alba Mill.

\section{ARECACEAE}

Sabal mexicana Mart.

Sabal yapa C. Wright ex Becc.

BIGNONIACEAE

Tabebuia rosea (Bertol.) DC.

BURSERACEAE

Bursera simaruba (L.) Sarg.

CAPPARACEAE

Quadrella isthmensis (Eichler) Hutch. ssp. mexicana X. Cornejo \& H. H. Iltis

COMBRETACEAE

Laguncularia racemosa (L.) C. F. Gaertn.

EBENACEAE

Diospyros yucatanensis Lundell ssp. yucatanensis

Diospyros sp.

\section{EUPHORBIACEAE}

Gymnanthes lucida $\mathrm{Sw}$.

\section{FABACEAE}

Lysiloma latisiliquum (L.) Benth

Piscidia piscipula (L.) Sarg.

Pithecellobium sp.

\section{MALVACEAE}

Hampea trilobata Standl.

\section{MELIACEAE}

Swietenia macrophylla King

Trichilia sp.

\section{MORACEAE}

Ficus obtusifolia Kunth

Ficus sp.

PRIMULACEAE

Bonellia macrocarpa (Cav.) B. Stahl \& Kallersjo ssp. macrocarpa

\section{RHAMNACEAE}

Krugiodendron ferreum (Vahl) Urb.

\section{RHIZOPHORACEAE}

Rhizophora mangle L.

\section{SALICACEAE}

Zuelania guidonia (Sw.) Britton \& Millsp.

SAPINDACEAE

Melicoccus oliviformis Kunth ssp. oliviformis

\section{SAPOTACEAE}

Manilkara zapota (L.) P. Royen

Pouteria reticulata (Engl.) Eyma ssp. reticulata

Sideroxylon americanum (Mill.) T.D. Penn. 